\title{
International collaboration to assess the risk of Guillain Barré Syndrome following Influenza A (H1N1) 2009 monovalent vaccines ${ }^{\text {th }}$
}

\author{
Caitlin N. Dodd ${ }^{\mathrm{a}, *}$, Silvana A. Romio ${ }^{\mathrm{b}}$, Steven Black ${ }^{\mathrm{c}}$, Claudia Vellozzi $^{\mathrm{d}}$, Nick Andrews ${ }^{\mathrm{e}}$, \\ Miriam Sturkenboom $^{\mathrm{b}}$, Patrick Zuber ${ }^{\mathrm{f}}$, Wei Hua ${ }^{\mathrm{g}}$, Jan Bonhoeffer ${ }^{\mathrm{h}, \mathrm{i}}$, Jim Buttery ${ }^{\mathrm{j}}$, \\ Nigel Crawford ${ }^{\mathrm{k}}$, Genevieve Deceuninck ${ }^{1}$, Corinne de Vries $^{\mathrm{m}}$, Philippe De Wals ${ }^{1}$, \\ M. Victoria Gutierrez- Gimeno ${ }^{\mathrm{n}}$, Harald Heijbel $^{ }$, Hayley Hughes $^{\mathrm{p}}$, Kwan Hur ${ }^{\mathrm{q}}$, \\ Anders Hviid $^{\mathrm{r}}$, Jeffrey Kelman ${ }^{\mathrm{s}}$, Tehri Kilpi ${ }^{\mathrm{t}}$, S.K. Chuang ${ }^{\mathrm{u}}$, Kristine Macartney ${ }^{\mathrm{v}}$, \\ Melisa Rett ${ }^{\mathrm{w}}$, Vesta Richardson Lopez-Callada ${ }^{\mathrm{x}}$, Daniel Salmon ${ }^{\mathrm{y}, \mathrm{z}}$, \\ Francisco Gimenez Sanchez $^{\text {aa }}$, Nuria Sanz ${ }^{\text {bb }}$, Barbara Silverman ${ }^{c c}$, Jann Storsaeter ${ }^{\text {dd }}$, \\ Umapathi Thirugnanam $^{\mathrm{ee}}$, Nicoline van der Maas ${ }^{\mathrm{ff}}$, Katherine Yih ${ }^{\mathrm{w}}$, Tao Zhang ${ }^{\mathrm{gg}}$, \\ Hector Izurieta $^{\mathrm{g}}$, the Global H1N1 GBS Consortium ${ }^{1}$
}

a Department of Biostatistics and Epidemiology, Cincinnati Children's Hospital Medical Center, Cincinnati, OH, United States

${ }^{\mathrm{b}}$ Department of Medical Informatics, Erasmus University Medical Center, Rotterdam, The Netherlands

' Department of Global Child Health, Cincinnati Children's Hospital Medical Center, Cincinnati, OH, United States

d Centers for Disease Control and Prevention, Atlanta, GA, United States

e Health Protection Agency, London, United Kingdom

f Department of Immunizations, Vaccines and Biologicals (IVB), World Health Organization, Genève, Switzerland

$\mathrm{g}$ Office of Biostatistics and Epidemiology, Center for Biologics Evaluation and Research, Food and Drug Administration, Rockville, MD, United States

h Brighton Collaboration Foundation, Basel, Switzerland

i University Children's Hospital, Basel, Switzerland

${ }^{\mathrm{j}}$ Murdoch Children's Research Institute, Dept of Paediatrics, Monash University, Victoria, Australia

${ }^{\mathrm{k}}$ The Department of Paediatrics, University of Melbourne, Melbourne, Australia

${ }^{1}$ Public Health Research Unit, Quebec University Hospital Research Centre, Quebec City, Quebec, Canada

m Department of Pharmacy \& Pharmacology, University of Bath, Bath, United Kingdom

${ }^{n}$ Vaccine Department, Centre of Public Health Research (CSISP-FISABIO), Valencia, Spain

o Swedish Institute for Infectious Disease Control, Lund, Sweden

P Department of Defense, Washington, DC, United States

q Center for Medication Safety, Pharmacy Benefit Management Services, Hines Veterans Affairs Hospital, Hines, IL, United States

${ }^{\mathrm{r}}$ Department of Epidemiology Research, Statens Serum Institute, Copenhagen, Denmark

${ }^{s}$ Center for Drug and Health Plan Choice, Centers for Medicare and Medicaid Services, Baltimore, MD, United States

${ }^{\mathrm{t}}$ National Institute for Health and Welfare, Helsinki, Finland

"Centre for Health Protection, Department of Health, Hong Kong

${ }^{v}$ Children's Hospital at Westmead, Westmead, New South Wales, Australia

${ }^{w}$ Department of Population Medicine, Harvard Medical School and Harvard Pilgrim Health Care Institute, Boston, MA, United States

x National Center for Child and Adolescent Health, Mexico City, Mexico, Mexico

y National Vaccine Program Office, Office of the Assistant Secretary for Health, U.S Department of Health and Human Services, Washington, DC, United States

${ }^{z}$ Department of International Health and Institute for Vaccine Safety, Johns Hopkins Bloomberg School of Public Health, Baltimore, MD, United States

aa Pediatrics Department, Torrecárdenas Hospital, Almería, Spain

${ }^{\mathrm{bb}}$ Hospital Clinic i Provincial de Barcelona (SCReN), Barcelona, Spain

cc Maccabi Healthcare Services, Tel Aviv, Israel

dd Norwegian Institute of Public Health, Oslo, Norway

ee National Neuroscience Institute, Singapore

ff RIVM, Bilthoven, The Netherlands

${ }^{\mathrm{gg}}$ Department of Epidemiology, School of Public Health, Fudan University, Key Laboratory of Public Health Safety, Ministry of Education, Shanghai, PR China

\footnotetext{
Abbreviations: CSF, cerebrospinal fluid; GP, general practitioner; MCRI, Murdoch Children's Research Institute; Maccabi, Maccabi Health Maintenance Organization; ICPI,

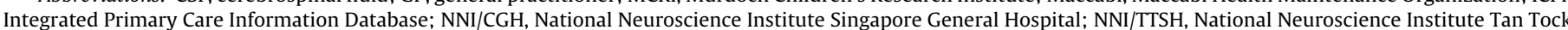

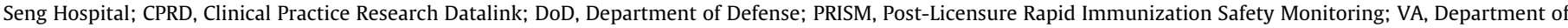
Veterans Affairs; VAESCO, Vaccine Adverse Event Surveillance and Communication Consortium; VSD, Vaccine Safety Datalink.

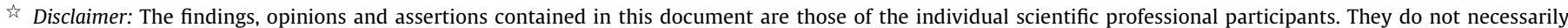
represent the official position of any of the participant's organizations (e.g., government, university, or corporations).

* Corresponding author. Tel.: +31645574714; fax: +31107044722.

E-mail addresses: c.dodd@erasmusmc.nl, caitlinndodd@gmail.com (C.N. Dodd).

1 See Appendix A for the group of Global H1N1 GBS Consortium.
} 


\section{A R T I C L E I N F O}

\section{Article history:}

Received 22 April 2013

Received in revised form 24 May 2013

Accepted 5 June 2013

Available online 14 June 2013

\section{Keywords:}

Guillain Barré Syndrome (GBS)

Monovalent H1N1 Vaccine (H1N1)

Self-controlled case-series method (SCCS)

International

Adjuvant

Adverse events following immunization (AEFI)

\section{A B S T R A C T}

Background: The global spread of the 2009 novel pandemic influenza A(H1N1) virus led to the accelerated production and distribution of monovalent 2009 Influenza A (H1N1) vaccines (pH1N1). This pandemic provided the opportunity to evaluate the risk of Guillain-Barre syndrome (GBS), which has been an influenza vaccine safety concern since the swine flu pandemic of 1976, using a common protocol among high and middle-income countries. The primary objective of this project was to demonstrate the feasibility and utility of global collaboration in the assessment of vaccine safety, including countries both with and without an established infrastructure for vaccine active safety surveillance. A second objective, included $a$ priori, was to assess the risk of GBS following pH1N1 vaccination.

Methods: The primary analysis used the self-controlled case series (SCCS) design to estimate the relative incidence (RI) of GBS in the 42 days following vaccination with pH1N1 vaccine in a pooled analysis across databases and in analysis using a meta-analytic approach.

Results: We found a relative incidence of GBS of $2.42(95 \% \mathrm{CI} 1.58-3.72)$ in the 42 days following exposure to $\mathrm{pH} 1 \mathrm{~N} 1$ vaccine in analysis of pooled data and 2.09 (95\% CI 1.28-3.42) using the meta-analytic approach. Conclusions: This study demonstrates that international collaboration to evaluate serious outcomes using a common protocol is feasible. The significance and consistency of our findings support a conclusion of an association between $2009 \mathrm{H} 1 \mathrm{~N} 1$ vaccination and GBS. Given the rarity of the event the relative incidence found does not provide evidence in contradiction to international recommendations for the continued use of influenza vaccines.

(c) 2013 Elsevier Ltd. All rights reserved.

\section{Introduction}

Assessment of vaccine safety post-licensure requires welldesigned epidemiological studies, which can be challenging for many countries due to scarcity of available data. Therefore, spontaneous reporting systems are more commonly used for postmarketing safety monitoring [1]. Traditionally, vaccines have been manufactured and introduced in the United States (US) and Europe before introduction in other countries, hence US and European vaccine safety monitoring capacity has served the global need to evaluate the safety of new vaccines [1]. However, vaccines are now being manufactured and introduced in several countries outside the US and Europe [2], requiring the development of vaccine safety monitoring systems globally to assure the safety of the world's vaccine supply and maintain trust in immunization programs. International vaccine safety collaborations can help build vaccine safety monitoring infrastructure and capacity and provide a means to assess rare adverse events following immunization (AEFI) in countries that now have limited capacity [3].

To demonstrate that international collaboration is feasible for vaccine safety studies to investigate rare, serious and clinically complex AEFI, a group of vaccine safety researchers conducted a proof of concept collaborative vaccine safety study using a standard protocol [4-6]. A steering group ${ }^{2}$ from the World Health Organization (WHO), United States Food and Drug Administration (FDA) and Centers for Disease Control and Prevention (CDC), European CDC, Erasmus Medical Center, Cincinnati Children's Hospital, and the Brighton Collaboration [7], provided standardized methods and definitions for a study that included investigators from Australia, Canada, China, Denmark, Finland, France, Israel, Mexico, The Netherlands, Norway, Singapore, Spain, Sweden, the United Kingdom, and the United States.

The global spread of the 2009 novel pandemic influenza A (H1N1) virus [8] led to the accelerated production of monovalent 2009 Influenza A (H1N1) vaccines (pH1N1) by manufacturers in the Americas, Europe, and Asia [9]. Rapid and extensive vaccine administration was implemented worldwide. This pandemic provided the opportunity to evaluate the risk of Guillain-Barré syndrome (GBS), an acute polyradiculoneuropathy, following receipt of these vaccines using a common protocol among high and middle-income

\footnotetext{
2 Steven Black, Caitlin Dodd (Cincinnati Children's Hospital), Hector Izurieta (FDA), Patrick Zuber (WHO).
}

countries and to assess the feasibility of this collaborative effort [10]. Several factors contributed to choosing this vaccine and this adverse event (GBS) to test the new consortium: First, GBS has been an influenza vaccine safety concern since 1976 , when an elevated risk of GBS was identified following the "swine-flu" influenza vaccine [11]; second, case definitions and classifications for GBS are available, providing a tool for standardized assessment across sites [12]; third, since almost all GBS cases are hospitalized, unbiased case ascertainment could be achieved using hospital databases; and finally, since GBS is rare, assessment of risk would benefit from the increased sample size and statistical power that could result from an international collaboration.

The primary objective of this project was to demonstrate the feasibility and utility of global collaboration in the assessment of vaccine safety, including countries both with and without an established infrastructure for vaccine safety active surveillance. A second objective, included a priori, was to assess the relative risk of GBS following $\mathrm{pH} 1 \mathrm{~N} 1 \mathrm{vaccination}$.

\section{Methods}

We chose the self-controlled case series (SCCS) design [13] to estimate the relative incidence (RI) of GBS in the 42 days following vaccination with $\mathrm{pH} 1 \mathrm{~N} 1$ vaccine. We chose this case-only analytic approach because it can be implemented in populations with varying levels of infrastructure for conducting epidemiologic studies; specifically, it does not require the availability of accurate population denominators which are difficult to obtain in many countries $[9,10]$. The case series approach includes only individuals who experienced the event of interest (GBS) in the analysis. Each individual's person-time during follow-up is divided into predefined vaccine exposed and non-exposed periods. Each GBS case then falls into a risk or non-risk window and contributes exposed and nonexposed time. Unvaccinated GBS cases contribute to the estimation of other time-varying covariates such as seasonality. Data are analyzed by conditional Poisson regression. The SCCS design requires that cases be ascertained completely and in an unbiased manner and that the probability of exposure is not affected by occurrence of the event of interest. Apart from its intrinsic resource efficiency, this design also controls for measured or unmeasured within-person non-time dependent confounding characteristics, including demographics and chronic co-morbid conditions, genetic susceptibility, and others [10]. 
Table 1

Database inclusion and exclusion in primary and sensitivity analyses by Country.

\begin{tabular}{|c|c|c|c|}
\hline Country & Database & Criteria for exclusion & \\
\hline \multicolumn{4}{|l|}{ Excluded databases } \\
\hline France & & \multicolumn{2}{|l|}{ Patient Consent Required (potential bias) } \\
\hline Israel & Maccabi & \multicolumn{2}{|c|}{ Brighton Collaboration criteria not provided } \\
\hline Mexico & Mexico City & \multicolumn{2}{|c|}{ Relative incidence found to be an outlier compared to all other study site relative incidence findings } \\
\hline & Mexican States & \multicolumn{2}{|c|}{ Data were obtained solely from a specialist network (potential bias) } \\
\hline Norway & & \multicolumn{2}{|c|}{ Data were obtained solely from a specialist network (potential bias) } \\
\hline Sweden & & \multicolumn{2}{|c|}{ Patient consent required with potential bias } \\
\hline Country & Database & Analyses in which database is included & Criteria for exclusion where applicable \\
\hline \multicolumn{4}{|l|}{ Included databases } \\
\hline \multirow[t]{6}{*}{ Australia } & Adelaide & - Primary analysis & \\
\hline & & - All sensitivity analyses & \\
\hline & MCRI $^{\mathrm{a}}$ & - Primary analysis & \\
\hline & & - All sensitivity analyses & \\
\hline & Sydney & - Primary analysis & \\
\hline & & - All sensitivity analyses & \\
\hline \multirow[t]{2}{*}{ Canada } & Quebec & - Primary analysis & \\
\hline & & - All sensitivity analyses & \\
\hline \multirow[t]{3}{*}{ China } & Hong Kong & - Primary analysis & \\
\hline & & - All sensitivity analyses & \\
\hline & Shanghai & - Analyses through Brighton 4A & All reported cases were Brighton level 4 or $4 \mathrm{~A}$ \\
\hline \multirow[t]{2}{*}{ Denmark } & & - Primary analysis & \\
\hline & & - All sensitivity analyses & \\
\hline \multirow[t]{2}{*}{ Finland } & & - Primary analysis & \\
\hline & & - All sensitivity analyses & \\
\hline \multirow[t]{2}{*}{ The Netherlands } & $\mathrm{IPCl}^{\mathrm{a}}$ & - Primary analysis & \\
\hline & & - All sensitivity analyses & \\
\hline \multirow[t]{3}{*}{ Singapore } & $\mathrm{NNI} / \mathrm{CGH}^{\mathrm{a}}$ & - Primary analysis & \\
\hline & & - All sensitivity analyses & \\
\hline & $\mathrm{NNI} / \mathrm{TTSH}^{\mathrm{a}}$ & - Analyses through Brighton $4 \mathrm{~A}$ & All reported cases were Brighton level 4 or $4 \mathrm{~A}$ \\
\hline \multirow[t]{5}{*}{ Spain } & Almeria & - Analyses through Brighton $4 \mathrm{~A}$ & All reported cases were Brighton level 4 or $4 \mathrm{~A}$ \\
\hline & Barcelona & - Primary analysis & \\
\hline & & - All sensitivity analyses & \\
\hline & Valencia & - Primary analysis & \\
\hline & & - All sensitivity analyses & \\
\hline The United Kingdom & $\mathrm{CPRD}^{\mathrm{a}}$ & - Analyses through Brighton $4 \mathrm{~A}$ & All reported cases were Brighton level 4 or $4 \mathrm{~A}$ \\
\hline \multirow[t]{6}{*}{ The United States } & $\mathrm{DoD}^{\mathrm{a}}$ & - Primary analysis & \\
\hline & & - All sensitivity analyses & \\
\hline & Medicare & - Vaccinated-cases only analysis, excluded from all others & Database contained only cases post-vaccination \\
\hline & PRISM $^{\mathrm{a}}$ & - Vaccinated-cases only analysis, excluded from all others & Database contained only cases post-vaccination \\
\hline & $\mathrm{VA}^{\mathrm{a}}$ & - Vaccinated-cases only analysis, excluded from all others & Database contained only cases post-vaccination \\
\hline & $\mathrm{VSD}^{\mathrm{a}}$ & - Vaccinated-cases only analysis, excluded from all others & Database contained only cases post-vaccination \\
\hline
\end{tabular}

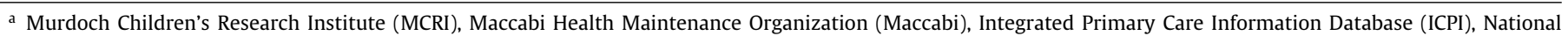

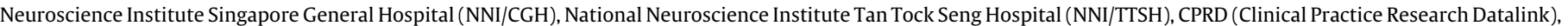
Department of Defense (DoD), Post-Licensure Rapid Immunization Safety Monitoring (PRISM), Department of Veterans Affairs (VA), Vaccine Safety Datalink (VSD).

\subsection{Study population}

As shown in Table 1, 15 countries with available data and willingness to participate contributed data for this study: cases that met inclusion criteria for this study from Australia, Canada, China, Israel, Mexico, Singapore, Spain, and the United States and from the European Vaccine Adverse Event Surveillance and Communication (VAESCO) consortium [14] (http://vaesco.net) (Denmark, Finland, France, The Netherlands, Norway, Sweden, and the United Kingdom) were included. Australian data were provided by hospitals in the state of Victoria (including Melbourne), Sydney, Perth, and Adelaide. Canadian data were provided from the entire province of Quebec; Chinese cases were contributed by sentinel hospitals in Hong Kong and Shanghai. Israeli data were provided by Maccabi, a national health maintenance organization (HMO) and Mexican data were contributed from Mexico City and surrounding rural areas. Singapore data were provided from one rural and one central hospital. Spanish data were provided by hospitals in Almeria, Barcelona, and Valencia. US data were contributed from the Department of Defense (DoD), the Department Veterans Affairs (VA), the Vaccine Safety Datalink (combined hospitalization and vaccination data from a collaboration of 8 health care organizations),
Medicare, and the Post-Licensure Rapid Immunization Safety Monitoring (PRISM) Program [15,16].

\subsection{Case ascertainment and classification}

The specific method of case ascertainment varied from country to country with some countries identifying potential cases through administrative databases whereas other countries reviewed hospital discharge logs manually (Table 2). Databases from all US sites other than the DoD contained only vaccinated cases and were limited to post-vaccination follow up time. Each site independently defined an observation period, ranging from 4 to 18 months, during which cases were obtained. The number of medical records requested and reviewed was not reported by the participating sites. Countries that did not identify and classify cases using a procedure compatible with the common protocol were excluded from this study (Table 1). For countries in which cases were ascertained through active surveillance, only those cases with verified hospital admission were included.

Diagnostic codes for GBS (ICD-9 code of 357.0, ICD-10 code of G61.0, or Read codes F370*) were used to identify potential cases for review. Cases identified exclusively through specialty 
Table 2

Characteristics of databases included in primary or sensitivity analyses by country.

\begin{tabular}{|c|c|c|c|c|c|}
\hline Country & Database & Dates of observation & Number of cases & Case ascertainment & Vaccination status ascertainment \\
\hline \multirow[t]{3}{*}{ Australia } & Adelaide & $9 / 30 / 2009-9 / 30 / 2010$ & 1 & $\begin{array}{l}\text { Administrative database, active } \\
\text { prospective surveillance }\end{array}$ & $\begin{array}{l}\text { Vaccine Registry, Self-Report, } \\
\text { Outpatient Chart Review }\end{array}$ \\
\hline & $\mathrm{MCRI}^{\mathrm{a}}$ & $9 / 30 / 2009-9 / 30 / 2010$ & 54 & $\begin{array}{l}\text { Administrative database, active } \\
\text { prospective surveillance }\end{array}$ & $\begin{array}{l}\text { Vaccine Registry, Self-Report, } \\
\text { Outpatient Chart Review }\end{array}$ \\
\hline & Sydney & $9 / 30 / 2009-9 / 30 / 2010$ & 5 & $\begin{array}{l}\text { Administrative database, active } \\
\text { prospective surveillance }\end{array}$ & $\begin{array}{l}\text { Vaccine Registry, Self-Report, } \\
\text { Outpatient Chart Review }\end{array}$ \\
\hline Canada & Quebec & $10 / 13 / 2009-3 / 31 / 2010$ & 80 & $\begin{array}{l}\text { Administrative database, active } \\
\text { prospective surveillance }\end{array}$ & Vaccine registry \\
\hline \multirow[t]{2}{*}{ China } & Hong Kong & $12 / 21 / 2009-6 / 30 / 2010$ & 20 & Hospital log review & $\begin{array}{l}\text { Outpatient Chart Review, } \\
\text { Self-Report }\end{array}$ \\
\hline & Shanghai & $1 / 1 / 2009-7 / 1 / 2010$ & 22 & Administrative database & Outpatient Chart Review \\
\hline Denmark & & $11 / 1 / 2009-11 / 1 / 2010$ & 31 & $\begin{array}{l}\text { National Patient Register using } \\
\text { primary discharge diagnoses }\end{array}$ & Vaccine Registry \\
\hline Finland & & $11 / 1 / 2009-11 / 1 / 2010$ & 29 & $\begin{array}{l}\text { Hospital discharge and hospital } \\
\text { outpatient records, primary } \\
\text { diagnoses }\end{array}$ & Vaccine registry \\
\hline The Netherlands & $\mathrm{IPCl}^{\mathrm{a}}$ & $11 / 1 / 2009-11 / 1 / 2010$ & 80 & $\begin{array}{l}\text { Identified prospectively through } \\
\text { neurologists }\end{array}$ & $\mathrm{GP}^{\mathrm{b}}$ medical record \\
\hline & & & & $\begin{array}{l}\text { Completeness verified } \\
\text { retrospectively against claims } \\
\text { codes in each hospital. }\end{array}$ & \\
\hline \multirow[t]{2}{*}{ Singapore } & $\mathrm{NNI} / \mathrm{CGH}^{\mathrm{a}}$ & $11 / 5 / 2009-8 / 31 / 2010$ & 6 & Administrative Database & $\begin{array}{l}\text { Outpatient Chart Review, } \\
\text { Self-Report }\end{array}$ \\
\hline & $\mathrm{NNI} / \mathrm{TTSH}^{\mathrm{a}}$ & $11 / 5 / 2009-8 / 31 / 2010$ & 13 & Administrative Database & Hospital Medical Records \\
\hline \multirow[t]{3}{*}{ Spain } & Almeria & $11 / 1 / 2009-4 / 30 / 2010$ & 8 & Administrative Database & Outpatient Chart Review \\
\hline & Barcelona & $11 / 1 / 2009-4 / 30 / 2010$ & 14 & Administrative Database & Outpatient Chart Review \\
\hline & Valencia & $11 / 1 / 2009-4 / 30 / 2010$ & 10 & Administrative Database & Vaccine Registry \\
\hline The United Kingdom & $\mathrm{CPRD}^{\mathrm{a}}$ & $11 / 1 / 2009-11 / 1 / 2010$ & 40 & Automated GP records & $\mathrm{GP}^{\mathrm{b}}$ records \\
\hline \multirow[t]{5}{*}{ The United States } & $\mathrm{DoD}^{\mathrm{a}}$ & $11 / 1 / 2009-4 / 30 / 2010$ & 6 & $\begin{array}{l}\text { Administrative Database } \\
\text { Electronic Medical Records }\end{array}$ & Vaccine Registry \\
\hline & Medicare & $\begin{array}{l}11 / 1 / 2009- \\
4 / 30 / 2010\end{array}$ & 39 & $\begin{array}{l}\text { Administrative Database } \\
\text { Chart Review }\end{array}$ & Administrative Database \\
\hline & PRISM $^{\mathrm{a}}$ & $10 / 22 / 2009-8 / 7 / 2010$ & 8 & $\begin{array}{l}\text { Vaccine Registries and Claims } \\
\text { Databases }\end{array}$ & Electronic Medical Claims \\
\hline & $\mathrm{VA}^{\mathrm{a}}$ & $11 / 1 / 2009-4 / 30 / 2010$ & 2 & $\begin{array}{l}\text { Administrative Database } \\
\text { Electronic Medical Records }\end{array}$ & $\begin{array}{l}\text { Vaccine Registry and } \\
\text { Administrative database }\end{array}$ \\
\hline & $\mathrm{VSD}^{\mathrm{a}}$ & $8 / 1 / 2009-4 / 30 / 2010$ & 11 & $\begin{array}{l}\text { Administrative Database } \\
\text { Electronic Medical Records }\end{array}$ & $\begin{array}{l}\text { Vaccine Registry and } \\
\text { Administrative database. }\end{array}$ \\
\hline
\end{tabular}

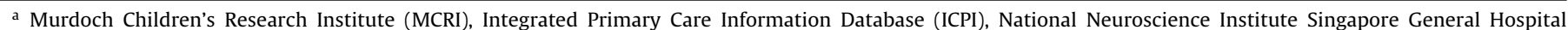

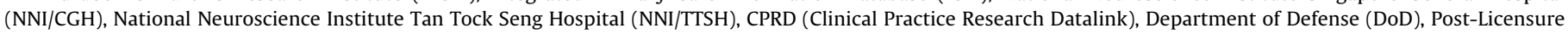
Rapid Immunization Safety Monitoring (PRISM), Department of Veterans Affairs (VA), Vaccine Safety Datalink (VSD)

b General practitioner.

network reporting or through other passive reporting method were excluded. The specific method of case ascertainment used and number of cases identified are shown in Table 2.

All cases were classified locally according to Brighton Collaboration criteria [12] (Table 3). All cases meeting Brighton level 1, 2, 3 were considered confirmed and included in the primary analysis. A secondary analysis also included Brighton categories 4 and $4 \mathrm{~A}$. Category $4 \mathrm{~A}$ was specifically defined for this international study and included cases diagnosed by a neurologist but for whom the medical chart did not provide sufficient information for the study reviewers to classify the case according to Brighton Collaboration criteria (Table 3).

Table 3

Brighton collaboration case definition for Guillain-Barré Syndrome [12]. ${ }^{\mathrm{a}}$

\begin{tabular}{|c|c|}
\hline Level & Requirements \\
\hline 1 & Clinical, electrophysiological, AND cerebrospinal fluid (CSF) data \\
\hline 2 & $\begin{array}{l}\text { Clinical, data and electrophysiological OR cerebrospinal fluid (CSF) } \\
\text { data }\end{array}$ \\
\hline 3 & Clinical data \\
\hline 4 & $\begin{array}{l}\text { Information available is insufficient for levels } 1-3 \text {, but no other } \\
\text { diagnosis is apparent or warranted. }\end{array}$ \\
\hline $4 \mathrm{~A}$ & $\begin{array}{l}\text { Diagnosis was made by a neurologist, insufficient diagnostic data } \\
\text { available in the medical chart (adopted specifically for this study) }\end{array}$ \\
\hline
\end{tabular}

\footnotetext{
a Levels 1-3 only used in the primary analysis, levels $1-4 \mathrm{~A}$ used in sensitivity analyses.
}

\subsection{Vaccination status}

Vaccination status was obtained through automated immunization registries or databases when available or through a review of the patient's vaccination record where it was not. In some cases, receipt of vaccine was obtained through self-report and subsequently verified in the vaccination record. The method used for each participating site is shown in Table 2 . All subjects had presence or absence of pH1N1 vaccine recorded with the date of exposure. Patients for whom no record of seasonal influenza vaccination were available were considered unexposed to the seasonal vaccine Table 4.

\subsection{Covariates}

Presence or absence of risk factors for GBS, including preceding gastrointestinal infections and respiratory infections, were collected for the 30 days prior to diagnosis through chart review or recall by the subject when chart review was not possible. While a standard abstraction form was not used, a standard case report form was used to record all data used in analysis. Since presence or absence of the potential infective episodes in the 30 days preceding GBS rather than exact dates of the episodes was collected, these infections could not be controlled for in the analysis but could be studied as potential effect modifiers. To control for circulation of the pandemic influenza virus we used seasonality as a proxy. 
Table 4

Number and characteristics of Guillain-Barré syndrome cases and relative incidence following pH1N1 vaccination by database from ten countries.

\begin{tabular}{|c|c|c|c|c|c|c|c|c|}
\hline Country & Database & $\begin{array}{l}\text { Number of } \\
\text { cases }\end{array}$ & $\begin{array}{l}\text { Number of pH1N1 }{ }^{\mathrm{b}} \\
\text { exposed cases }\end{array}$ & $\begin{array}{l}\text { Number of cases meeting } \\
\text { Brighton Criteria 1-4A }\end{array}$ & $\begin{array}{l}\text { Number of exposed cases } \\
\text { meeting Brighton Criteria 1-4A }\end{array}$ & $\begin{array}{l}\text { Age } \\
\left.\text { (mean, } \mathrm{SD}^{\mathrm{c}}\right)\end{array}$ & $\begin{array}{l}\text { Sex }=M \\
\text { (frequency, \%) }\end{array}$ & $\begin{array}{l}\text { Database-specific GBS }{ }^{d} \mathrm{RI}^{\mathrm{e}} \\
\left(\mathrm{CI}^{\mathrm{f}}\right) \text {, Brighton Levels } 1-4 \mathrm{~A}\end{array}$ \\
\hline \multirow[t]{3}{*}{ Australia } & Adelaide & 1 & 0 & 1 & 0 & $4\left(\mathrm{NA}^{\mathrm{g}}\right)$ & $1(100 \%)$ & NA \\
\hline & $\mathrm{MCRI}^{\mathrm{h}}$ & 54 & 10 & 54 & 10 & $49.2(23.9)$ & $30(56 \%)$ & $2.10(0.40,11.05)$ \\
\hline & Sydney & 5 & 0 & 5 & 0 & $5.8(4.7)$ & $3(60 \%)$ & NA \\
\hline Canada & Quebec & 80 & 43 & 80 & 43 & $49.5(21.9)$ & $55(69 \%)$ & $1.45(0.70,3.00)$ \\
\hline \multirow[t]{2}{*}{ China } & Hong Kong & 20 & 5 & 20 & 5 & $57.8(13.3)$ & $12(60 \%)$ & $0.88(0.07,11.32)$ \\
\hline & Shanghai & 22 & 0 & 22 & 0 & $42.0(18.1)$ & $15(68 \%)$ & NA \\
\hline Denmark & & 31 & 4 & 31 & 4 & $49.2(20.2)$ & $14(45.2)$ & $4.08(0.48,34.83)$ \\
\hline Finland & & 29 & 13 & 29 & 13 & $54.4(20.8)$ & $12(41.4)$ & $2.59(0.77,8.68)$ \\
\hline The Netherlands & $\mathrm{IPCI}^{\mathrm{h}}$ & 80 & 29 & 79 & 28 & $45.0(20.8)$ & $32(40.0)$ & $2.81(1.07,7.34)$ \\
\hline \multirow[t]{2}{*}{ Singapore } & $\mathrm{NNI} / \mathrm{CGH}^{\mathrm{h}}$ & 6 & 1 & 6 & 1 & $36.3(16.9)$ & $5(83 \%)$ & $3.60 \times 10^{9}(0$, infinity $)$ \\
\hline & $\mathrm{NNI} / \mathrm{TTSH}^{\mathrm{h}}$ & 13 & 2 & 13 & 2 & $54.9(16.7)$ & $9(69 \%)$ & $3.60 \times 10^{9}(0$, infinity $)$ \\
\hline \multirow[t]{3}{*}{ Spain } & Almeria & 8 & 1 & 8 & 1 & $45.9(20.3)$ & $5(63 \%)$ & $1.27 \times 10^{9}(0$, infinity $)$ \\
\hline & Barcelona & 14 & 0 & 14 & 0 & $38.9(23.7)$ & $8(57 \%)$ & NA \\
\hline & Valencia & 10 & 0 & 10 & 0 & $47.8(22.8)$ & $6(60 \%)$ & NA \\
\hline The United Kingdom & $\mathrm{CPRD}^{\mathrm{h}}$ & 40 & 3 & 40 & 3 & $45.4(20.4)$ & $17(42.5)$ & $10.92(0.92,130.13)$ \\
\hline \multirow[t]{6}{*}{ The United States } & $\operatorname{DoD}^{\mathrm{h}}$ & 6 & 6 & 6 & 6 & $28.8(6.8)$ & $6(100 \%)$ & $8.39(0.73,97.00)$ \\
\hline & Databases w & raccinated cas & & & & & & \\
\hline & Medicare & 39 & 39 & 35 & 35 & $72.8(8.5)$ & $25(64 \%)$ & $2.04(0.99,4.20)$ \\
\hline & PRISM $^{\mathrm{h}}$ & 8 & 8 & 7 & 7 & $48(33.5)$ & $4(50 \%)$ & $2.27(0.44,11.77)$ \\
\hline & $\mathrm{VA}^{\mathrm{h}}$ & 2 & 2 & 1 & 1 & $60(12.7)$ & $2(100 \%)$ & NA \\
\hline & $\mathrm{VSD}^{\mathrm{h}}$ & 11 & 11 & 11 & 11 & $51.5(24.2)$ & $4(36 \%)$ & $3.78(0.92,15.61)$ \\
\hline
\end{tabular}

${ }^{\text {a }}$ Descriptive statistics are for all cases regardless of Brighton Collaboration Criteria unless otherwise specified.

b monovalent 2009(H1N1) A vaccines.

c Standard deviation.

d Guillain-Barré syndrome.

e Relative incidence.

f Confidence interval.

'Not applicable.

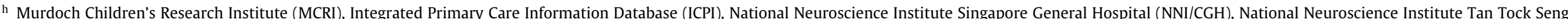

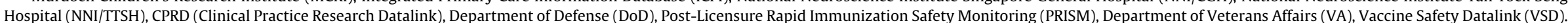


This was accomplished by defining the peak of influenza season for each site as the period during which $>15 \%$ of all surveillance influenza laboratory tests were positive, and estimating the relative incidence of GBS in this peak season. This produced time periods for each site defined either as "high influenza circulation" or "low influenza circulation". Although the seasonality of GBS is not strictly related to influenza infection, influenza surveillance data provided an efficient means by which to uniformly define seasonal periods across continents and hemispheres. This formulation allowed for a peak influenza season specific to each site while also allowing for a common estimate of the effect of seasonality across sites. Data to determine these periods of influenza circulation were obtained from publicly available governmental influenza surveillance, where available. For some sites, it was necessary to obtain these data from influenza surveillance conducted at the site.

\subsection{Data collection and sharing}

All data, with the exception of data from the VAESCO consortium, were uploaded to a secure WHO workspace where it was checked for quality and completeness by study group statisticians; VAESCO data were maintained by the VAESCO data management center at Erasmus Medical Center. All data was de-identified prior to submission. Institutional Review Board approval was obtained for those sites at which the study was not considered exempt. Inclusion and exclusion criteria as well as protocol and statistical analysis considerations were discussed on bi-weekly telephone conferences with all sites beginning in January, 2010.

\subsection{Analysis and statistical methods}

Data were analyzed using the SCCS method to investigate whether $\mathrm{pH} 1 \mathrm{~N} 1$ vaccination was associated with an increased risk of GBS during the pre-specified high-risk time window of days 1-42 post vaccination. This period of increased risk was chosen because of evidence from previous published studies [11]. We conducted two co-primary analyses: an analysis pooling all individuals across sites and an analysis using a meta-analytic approach in which estimates of rate ratios from each database were weighted based upon within and between-study errors and subsequently merged. While the pooled analysis provides more power, the meta-analytic approach is more conservative in its estimation as it weighs results from sites with less variability more heavily, thus providing greater assurance that outlying observations or sites with highly variable data will not bias the overall RI estimate. All analyses using the standard SCCS approach excluded the two weeks preceding vaccination from the background period to account for a possible healthy vaccinee effect [17], and controlled for seasonality as defined by periods of circulating influenza.

Only cases meeting Brighton Collaboration criteria level 1-3 [12] from databases which included pre and post-vaccination time were included in the primary analysis, which also included a timevarying covariate to assess the effect of seasonality. The date of diagnosis or hospitalization was used as the index date for GBS.

The standard SCCS method is only valid if the occurrence of an event (GBS in this case) does not alter the probability of subsequent exposure. This assumption may be violated since knowledge on the part of practitioners and patients regarding the a priori association between GBS and swine flu vaccine [11] may influence vaccination practices, and patients may forego or delay vaccination after GBS diagnosis [18]. For this reason, we also evaluated GBS risk using modifications of the standard SCCS approach to analyze data in which the event-dependent exposures assumption may have been violated. The first of these is the vaccinees-only approach, in which only vaccinated subjects are included and the observation window begins at the date of vaccination. The removal of non-vaccinated cases reduces power to estimate the effect of time-varying covariates such as seasonality [19]. The second modification is the pseudo-likelihood approach, a novel method which considers all cases (vaccinated or not) included in the analysis [20]. In this extension to the standard SCCS, we estimated risk under a counterfactual in which every vaccine exposure is treated as the last possible exposure for that subject. The inclusion of all cases during the entire observation period retains optimal power for the estimation of time-varying covariates [20]. The pseudo-likelihood approach was used to estimate the effect of pH1N1 vaccine with adjustment for seasonality. The vaccinated-cases only and pseudolikelihood approaches are equivalent when only one exposure is considered and no time-dependent covariates are included in the analysis. Because there was less than $20 \%$ difference between the pH1N1 vaccine-associated relative incidence estimates from the standard and pseudo-likelihood approaches (the difference decided a priori as evidence of bias from contraindication), all subsequent analyses were conducted using the standard approach.

A series of sensitivity analyses were conducted. To assess the effect of seasonal influenza vaccination, the exposure dates were included when known and subjects with missing data were assumed to be non-recipients of seasonal vaccine. We also assessed the risk window in more detail using days 1-7, 8-21, and 22-42 and estimated risks for each different window simultaneously within the same model. Subsequently, cases meeting Brighton criteria through level 4 and $4 \mathrm{~A}$ were included and an analysis using the date of onset rather than the date of diagnosis was conducted. An analysis limited to Brighton criteria levels 1 and 2 was also conducted. To understand possible effect modifiers and confounders, analyses stratified by sex, age category $(<5,5-9,10-18,19-49,50-64$, and $65+$ years), history of GBS, and presence of recent infections were also performed. To capitalize on the diversity of vaccine types and manufacturers in the data set, we also stratified by adjuvanted and non-adjuvanted vaccines.

In the meta-analytic approach, we adjusted for seasonality using month-long periods rather than seasonal peaks since, as data were not being pooled across databases, a common measure of seasonality was not necessary. Estimates of the exposure to $\mathrm{pH} 1 \mathrm{~N} 1$ vaccine in each database, considering only first dose as exposure of interest, were subsequently combined using a meta-analytic approach with a random effects model in which the estimate from each site is weighted by the inverse of its variance plus the variance of estimates between databases [21]. All analyses were conducted using SAS 9.2 (SAS Institute, Cary NC).

\section{Results}

\subsection{Pooled data analysis}

In the primary analysis of pooled data limited to Brighton Collaboration criteria levels 1-3 (Table 5), we found a RI of 2.86 (95\% CI 1.88-4.34). In country-specific analyses for the meta-analytic approach, analysis of the data contributed by the Mexico City database was found to have a very high RI of 39.19 (3.74, 410.41). When we excluded Mexican cases from the primary analysis, the estimate of the RI for the primary analysis decreased to 2.42 (1.58, 3.72) (Table 5). Based upon these results, cases from Mexico were excluded from all analyses along with cases from those databases with potential ascertainment bias, resulting in 10 countries contributing cases to the analysis data set (Table 1).

The vaccinated cases only approach produced an estimated RI of $2.37(1.47,3.85)$. The pseudo-likelihood approach produced a similar point estimate of $2.23(1.42,3.52)$ (Table 5$)$.

In sensitivity analyses, inclusion of cases through Brighton criteria levels 4 and $4 \mathrm{~A}$ increased the RI estimate from the 
Table 5

Relative incidence of GBS following pH1N1 vaccination in data pooled across twenty databases from ten countries.

\begin{tabular}{|c|c|c|c|c|c|}
\hline Analysis & Risk window(s) & Exclusions & $\begin{array}{l}\text { Brighton } \\
\text { criteria levels }\end{array}$ & $\begin{array}{l}\text { Relative } \\
\text { incidence }\end{array}$ & $\begin{array}{l}\text { Confidence } \\
\text { interval }\end{array}$ \\
\hline \multicolumn{6}{|l|}{ Primary analysis } \\
\hline $\begin{array}{l}\text { Standard self-controlled } \\
\text { case series (SCCS) }\end{array}$ & Days $1-42$ & - Databases (DBs) with vaccinated cases only & $1-3$ & 2.42 & $(1.58,3.72)$ \\
\hline \multicolumn{6}{|l|}{ Sensitivity analyses } \\
\hline Standard SCCS & Days 1-42 & - DBs with vaccinated cases only & $1-4 \mathrm{~A}$ & 2.83 & $(1.91,4.19)$ \\
\hline Standard SCCS & Days $1-42$ & - DBs with vaccinated cases only & $1-2$ & 2.34 & $(1.48,3.70)$ \\
\hline Standard SCCS & Days $1-42$ & $\begin{array}{l}\text { - DBs with vaccinated cases only } \\
\text { - Cases with reported URI or ILI in the } 30 \text { days before } \\
\text { diagnosis }\end{array}$ & $1-3$ & 2.88 & $(1.79,4.65)$ \\
\hline Standard SCCS & Days $1-42$ & $\begin{array}{l}\text { - DBs with vaccinated cases only } \\
\text { - Cases with reported GI in the } 30 \text { days before diagnosis }\end{array}$ & $1-3$ & 2.73 & $(1.75,4.26)$ \\
\hline Pseudo-likelihood & Days $1-42$ & - DBs with vaccinated cases only & $1-3$ & 2.23 & $(1.42,3.52)$ \\
\hline Pseudo-likelihood & Days $1-42$ & - DBs with vaccinated cases only & $1-4 \mathrm{~A}$ & 2.59 & $(1.72,3.90)$ \\
\hline Vaccinated cases only & Days $1-42$ & $\begin{array}{l}\text { - Unvaccinated cases } \\
\text { - Cases vaccinated after diagnosis }\end{array}$ & $1-3$ & 2.37 & $(1.47,3.85)$ \\
\hline Standard SCCS & $\begin{array}{l}\text { Days } 1-7 * \\
8-21^{*} \\
22-42^{*} \\
\text { *Modeled Simultaneously }\end{array}$ & • DBs with vaccinated cases Only & $1-3$ & $\begin{array}{l}2.61 \\
3.11 \\
1.91\end{array}$ & $\begin{array}{l}(1.17,5.84) \\
(1.77,5.47) \\
(1.07,3.42)\end{array}$ \\
\hline $\begin{array}{l}\text { Vaccinated cases only } \\
\text { adjuvanted }\end{array}$ & Days $1-42$ & $\begin{array}{l}\text { - Unvaccinated cases } \\
\text { - Cases vaccinated after diagnosis } \\
\text { - Non-adjuvanted vaccine recipients }\end{array}$ & $1-3$ & 1.88 & $(1.04,3.41)$ \\
\hline $\begin{array}{l}\text { Vaccinated cases only } \\
\text { Non-adjuvanted }\end{array}$ & Days 1-42 & $\begin{array}{l}\text { - Unvaccinated cases } \\
\text { - Cases vaccinated after diagnosis } \\
\text { - Adjuvanted vaccine recipients }\end{array}$ & $1-3$ & 2.97 & $(1.13,7.84)$ \\
\hline
\end{tabular}

primary analysis (Brighton criteria 1-3) using the standard SCCS and pseudo-likelihood approaches to $2.83(1.91,4.19)$ and 2.59 $(1.72,3.90)$, respectively. Limiting included cases to Brighton criteria levels 1 and 2 in analysis using the standard SCCS only slightly reduced the estimate to 2.34 (1.48, 3.70). Adjusting for seasonal influenza vaccine exposure led to no change in the pH1N1associated estimate, $2.57(1.68,3.93)$ ( $p$ value $v s$. primary analysis $\mathrm{RI}=0.85$ ) and found no increase in relative incidence associated with seasonal influenza vaccine exposure $[0.77(0.28,2.14)]$. Using recorded date of onset as opposed to the date of diagnosis as the index date produced almost no change, likely due to the fact that the risk interval is long (data not shown). The analyses for multiple risk periods following vaccination yielded estimates of 2.61 (1.27, $6.35), 3.11(2.18,6.46)$, and $1.91(1.31,3.98)$ for risk windows of $1-7$, 8-21 and 22-42 days following vaccination, respectively. Excluding subjects with a reported history of GBS led to a slightly reduced estimate of $2.27(1.47,3.51)$. Excluding patients with reported influenza like illness or upper respiratory illness in the 30 days before onset of GBS slightly increased the pH1N1-associated estimate to $2.88(1.79,4.65)$. The exclusion of those with reported gastrointestinal illness also increased the vaccine-associated estimate to $2.73(1.75,4.26)$.

In age-stratified analyses, using the standard SCCS showed that the RI in days 1-42 following exposure increased with age: children age $<19$ years, the RI was $0.73(0.16,3.46)$, adults age $19-49$ years, $R I=1.56(0.51,4.71)$, age $50-64$ years, $R I=2.78(1.36,5.68)$, and $4.30(2.18,8.50)$ in those 65 and older. These confidence intervals overlap, suggesting a trend rather than a statistically significant difference in relative incidence by age. In standard SCCS analysis stratified by sex, the estimated RI was slightly higher in males, 2.75 $(1.65,4.57)$ than in females, $2.34(1.09,5.04)$ although the difference was not statistically significant $(p=0.73)$.

The estimate of adjuvanted vaccines, performed using the vaccinated cases only approach, yielded a RI estimate of $1.88(1.03,3.41)$ while the non-adjuvanted estimate was higher at $2.97(1.13,7.84)$. This difference was not statistically significant $(p=0.43)$.

Because data on cases exposed to vaccines containing the MF-59 adjuvant were limited to one database, we were unable to reliably compare the RI associated with each of the two adjuvants.

\subsection{Meta-analytic approach}

Results from the meta-analytic approach were similar to those from the pooled analysis but the magnitude of the estimates was decreased (Table 6). The standard SCCS approach produced an estimate of $2.09(1.28,3.42)$ while the vaccinated cases only approach produced an estimate of $2.33(1.5,3.62)$. Analysis of adjuvanted and non-adjuvanted vaccines using the meta-analytic approach yielded RI estimates of $1.65(0.86,3.19)$ and $3.10(1.70,5.65)$, respectively. This difference between adjuvanted and non-adjuvanted exposures was not statistically significant $(p=0.16)$.

\section{Discussion}

We have shown that international collaboration to evaluate serious rare outcomes using a common protocol is feasible and offers some advantages compared to single country or site analyses. Because GBS following vaccination is very rare with reported rates between 0.04 and 0.17 cases per 100,000 vaccinations [22], this combined analysis included a much larger number of cases than any published single country analysis and allowed inclusion of data from sites that did not have enough cases for a site-specific analysis. This provided both increased power to evaluate the outcome but also sufficient power to conduct sub-analyses by vaccine type. Secondly, the availability of data from several countries allowed us to identify a site (Mexico), which had a RI of GBS following pH1N1 vaccine much higher than that at any other site; had the analysis been conducted only in Mexico, conclusions regarding the risk of GBS following vaccination could have been inappropriately generalized to other populations.

We have found an increased risk of GBS following receipt of $\mathrm{pH} 1 \mathrm{~N} 1$ influenza vaccine. This risk is consistent with the level of risk reported by others. Estimates from single-country studies ranged from 1.05 to 4.70 , the majority of which reported statistically significant increased risk [23-32]. Estimates were lower in studies of adjuvanted vaccines $(1.05-3.04)[25,31]$ than in non-adjuvanted vaccines (1.57-4.70) [26-30,32].

Because we knew a priori that both adjuvanted and nonadjuvanted vaccines would be used within our study population, 
Table 6

Relative incidence of GBS following pH1N1 vaccination in results from twenty databases in ten countries, pooled using a meta-analytic approach.

\begin{tabular}{|c|c|c|c|c|c|}
\hline Analysis & Risk window(s) & Exclusions & $\begin{array}{l}\text { Brighton } \\
\text { criteria levels }\end{array}$ & $\begin{array}{l}\text { Relative } \\
\text { incidence }\end{array}$ & $\begin{array}{l}\text { Confidence } \\
\text { interval }\end{array}$ \\
\hline Self-controlled case series (SCCS) & Days $1-42$ & - Databases (DBs) with vaccinated cases only & $1-3$ & 2.09 & $(1.28,3.42)$ \\
\hline Vaccinated cases only & Days $1-42$ & $\begin{array}{l}\text { - Unvaccinated cases excluded } \\
\text { - Cases vaccinated after diagnosis }\end{array}$ & $1-3$ & 2.33 & $(1.50,3.62)$ \\
\hline Vaccinated cases only adjuvanted & Days $1-42$ & $\begin{array}{l}\text { - Unvaccinated cases } \\
\text { - Cases vaccinated after diagnosis } \\
\text { - Non-adjuvanted vaccine recipients }\end{array}$ & $1-3$ & 1.65 & $(0.86,3.19)$ \\
\hline Vaccinated cases only non-adjuvanted & Days $1-42$ & $\begin{array}{l}\text { - Unvaccinated cases } \\
\text { - Cases vaccinated after diagnosis } \\
\text { - Adjuvanted vaccine recipients }\end{array}$ & $1-3$ & 3.10 & $(1.70,5.65)$ \\
\hline
\end{tabular}

we included a comparative analysis in our analysis plan. In all our primary and sensitivity analyses, the risk of GBS following administration of non-adjuvanted vaccines was significantly elevated. The increased risk found for adjuvanted vaccines was not as consistent. It was significantly elevated in our pooled analyses but became nonsignificant in the meta-analysis. Moreover, the point estimates of the risk for adjuvanted vaccines were consistently lower than those for non-adjuvanted vaccines in all our analyses. This preliminary finding is reassuring, given general concerns regarding the use of adjuvanted vaccines for influenza, and the fact that these vaccines use less influenza antigen per dose, a useful advantage for pandemic situations during which the amount of available antigen for vaccine production may initially be limited. We hypothesize that one possible explanation for the apparent (non-significant) risk difference between adjuvanted and non-adjuvanted vaccines is the higher amount of influenza antigen in non-adjuvanted vaccines, although other factors could have contributed. Another possible explanation is increased protection from influenza in those who have received adjuvanted vaccines [33] and a subsequent reduction in GBS due to influenza infection, which may have confounded our results. The trend described was seen for both MF-59 and AS03 adjuvanted vaccine. However, only one country in the study (The Netherlands) used MF-59 adjuvanted vaccine, so our ability to compare adjuvants was limited. While we believe our results to be reassuring, they are by no means definitive. Although the difference between the RI estimates for adjuvanted and non-adjuvanted vaccines are not different in our pooled analysis $(p=0.43)$ or in our meta-analysis ( $p=0.16$ ), the finding warrants further investigation.

Results obtained through the primary analysis (the standard self-controlled case series), the pseudo-likelihood approach, and vaccinated-cases only up to Brighton level 3 were very similar, indicating there was likely little bias introduced if a history of GBS impacted vaccination practices during the 2009-2010 season. Exclusion of Brighton level 3 cases produced very little change in the RI estimate, suggesting lack of a diagnostic bias in the absence of electrophysiological or cerebrospinal fluid data. Inclusion of cases meeting Brighton Criteria levels 4 and $4 \mathrm{~A}$ increased the relative incidence estimate. Given that in these cases the diagnosis could not be reliably verified, it is unclear if this increase reflects a more complete capture of true GBS cases or the inclusion of non-true cases occurring near the date of vaccination because of diagnosis bias. Future studies in which the time and resources for centralized adjudication are available may be able to answer this question.

Inclusion of the reported date of seasonal influenza vaccination led to little change in the estimate. This could be attributed to minimal risk associated with seasonal influenza vaccine [34], or to under-reporting or confusion regarding whether seasonal or pandemic vaccine that was received leading to misclassification bias. In the analysis of multiple risk periods following vaccination, the increased incidence in the pre-specified high risk period including days 8-21 supports the 1976 finding of highest risk in weeks 2-3 following vaccination [11]. It has been hypothesized that the risk peaks during this interval because this is when the humoral immune response to the vaccine is highest [35]. Previous findings of increased risk in males as well as increasing risk with increasing age were also supported in our analyses [36]. The background incidence of GBS has also been shown to be about 40\% higher in males than in females and to increase with increasing age [37].

It is interesting to note that the exclusion of those subjects who experienced influenza-like illness or gastrointestinal illness resulted in small, non-significant increases in vaccinationassociated relative incidence, with $p$ values $v s$. primary analysis $R I=0.59$ and 0.70 , respectively. While these non-significant findings appear to be inconsistent with those published by the VAESCO consortium [14] and other studies [27], it is important to note that the SCCS is a methodology based upon an underlying timeline. As we did not have data on dates of influenza-like illness or gastrointestinal illness and could not include these as time-varying covariates, exclusion of those with an infection in the 30 days before diagnosis likely excluded infection-induced cases which should have occurred at similar rates within and outside of the vaccineassociated risk window. This exclusion would therefore serve to increase the estimated relative incidence associated with pH1N1 vaccine exposure.

An additional finding in our study is the increased GBS risk following $\mathrm{pH} 1 \mathrm{~N} 1$ vaccination with the increased age of vaccine recipients. This may be the result of a lower immune response in older individuals [38] with consequent increased susceptibility to H1N1 infection and subsequent infection-induced GBS in this age group. It may also be related to higher pre-vaccination antibody titers in those previously exposed to H1N1-like viruses [39] potentially leading to greater immune response following vaccination. This possible age effect requires further investigation.

As we have indicated, database-specific analysis of the data contributed by Mexico City produced a very high RI associated with pH1N1 exposure. We hypothesize that this could be due to a longer period of H1N1 circulation in Mexico prior to vaccine introduction with a high likelihood of vaccinated individuals having already been exposed to the H1N1 wild type virus [40,41] which may have induced a greater immune response upon receipt of $\mathrm{pH} 1 \mathrm{~N} 1 \mathrm{vac}-$ cine due to already elevated antibody titers. Additionally, cases of confirmed H1N1 infection in Mexico tended to have more severe clinical presentation and to result in death more frequently as compared to other countries, perhaps indicating greater virulence of the virus in the early stages of the pandemic [40]. However, control for seasonality in the Mexican database did not reduce the pH1N1 vaccine associated RI estimate. Further studies to elucidate the reason for this much higher risk level in Mexico are warranted.

The meta-analytic approach for pooling of database-specific relative incidences weights those databases with a large degree of variation less heavily than those with less variation. Therefore, databases with only one or two exposed cases and consequently with large standard errors, have much less weight in the combined estimate of $2.09(1.28,3.42)$. While this estimate is attenuated 
through the weights applied in the meta-analysis, the relative incidence of GBS in the 42 days following vaccination remains significantly elevated with a confidence interval very similar to that of the pooled estimate of $2.42(1.58,3.72)$. Interestingly, results from stratification of adjuvanted and non-adjuvanted vaccines in the meta-analytic analyses produced a non-significant relative incidence for adjuvanted vaccines and an increased relative incidence for non-adjuvanted vaccines. This can be interpreted as evidence that the trend of increased risk in non-adjuvanted vaccines as opposed to adjuvanted vaccines is not being driven by a set of influential databases.

In this proof of concept, we have learned that international collaborative database studies to evaluate vaccine safety are feasible, even across continents. However, the requirement that participating sites have access to databases from which cases could be ascertained in an unbiased manner limited participation to high and middle income sites with existing infrastructure to conduct active surveillance. However, new vaccines are now being introduced either exclusively in the developing world or concurrently with their release in developed countries. In addition, some newer vaccines, such as the malaria vaccine currently in phase three trials in Africa [42], will mainly target the developing world. These changes indicate the need for improved vaccine safety assessment in low and middle income countries to ensure the safety of new vaccines.

Developing capacity outside of developed countries to evaluate vaccine safety signals that arise out of passive surveillance systems or from other sources is necessary both to assure the safety of the world's vaccine supply and also to prevent vaccine safety scares from undermining successful programs. The only low to middle income country (LMIC) remaining in the final analysis was China, evidencing a need to increase infrastructure in LMIC for ascertainment of vaccination status and adjudication of adverse events following vaccination. In addition, evaluation of very rare event can be facilitated by the increased statistical power that could be achieved through international collaboration.

Although the protocol was common among sites, the degree to which sites were able to review charts and ascertain important covariates such as infections varied from site to site. Future collaborative studies would benefit from centralized case adjudication, improved data quality control and closer supervision of data abstraction and case ascertainment.

This study had several limitations. While the results of the analyses stratified by presence or absence of an adjuvant are intriguing, the use of adjuvanted or non-adjuvanted vaccines was generally homogeneous within each country. Because of this homogeneity, it was not possible to estimate the difference between adjuvanted and non-adjuvanted vaccine associated relative incidence within the same population and it was not possible to separate the effect of the vaccine formulation from the unknown effect of the country and its associated characteristics. Also, the observed association may have been modified by infections, some of which are known to increase the risk of GBS. While we attempted to control for concomitant infections such as influenza like illness and gastrointestinal illness, it was not possible within the limitation of the current study to include these infections as time varying covariates, which would be the ideal approach in the self-controlled case series methodology. Controlling for such time varying covariates may have attenuated the observed associations, as shown in previous studies [43-45]. Additionally, the use of reported seasonal peaks of circulating influenza was a means of estimating seasonal effects across sites but may not have been as accurate as the standard approach of estimating fixed-length periods when analyzing data from one geographic location. Given budget and data-sharing constraints, case verification was performed by each site and quality control was performed on site; a pooled review by a single expert group was not conducted. In spite of the use of common criteria provided by the Brighton Collaboration, the adjudication process may have varied from site to site.

\section{Conclusion}

We have demonstrated that multinational studies are feasible and can provide a useful platform to evaluate future vaccine safety concerns especially for rare, serious events. We look forward to the development of a sustainable global infrastructure in both developed and developing countries to meet global needs. The finding of much higher risk in Mexico and our ability to contrast this risk with that found in other countries using data submitted under a common protocol is a strength of this multinational study and supports the need for international collaboration in vaccine safety monitoring.

The significance and consistency of our findings support a conclusion of an association between 2009 (H1N1) vaccination and GBS. Nonetheless, given the rarity of the event the relative incidence found suggests that the vaccine would be responsible for very few excess GBS cases. Although we are not able to estimate attributable risk using the SCCS methodology, we know from other studies that the background risk of GBS is approximately 0.9 [46] cases per one million individuals and that the relative risk associated with the 1976-77 swine influenza vaccination campaign was 7.60 [11]. A relative incidence of 2-3 following vaccination would mean approximately 1-2 excess cases per one million vaccinees. Due to this minimal increase in incidence, our findings do not provide evidence in contradiction of international recommendations for the continued use of influenza vaccines.

This large collaborative multinational study has made possible the generation of a number of new hypotheses related to possible differences in risk of vaccine-associated GBS by age and by the use of adjuvants, which will require further investigation.

\section{Role of the funding source}

Minimal funding was provided by the World Health Organization and by the US Food and Drug Administration for creation and maintenance of an online workspace as well as travel for group statisticians to conduct data analysis. No funding was provided to investigators for their participation.

\section{Conflicts of Interest}

Steven Black is a consultant for Novartis Vaccines and serves on Data and Safety Monitoring Boards for GlaxoSmithKline.

Nigel Crawford received CSL ltd. support for the investigator-led study from which Australian data was contributed.

Philippe De Wals has received research grant support for other work from GlaxoSmithKline.

Tehri Kilpi has received research grant support for other work from GlaxoSmithKline.

Miriam Sturkenboom is the head of a research unit that occasionally conducts research for pharmaceutical industries, always with the full freedom to publish. These include: Novartis, EliLilly, Boehringer Ingelheim and Pfizer. There is no involvement of them in this paper.

Tao Zhang has received research grant support for other work from Pfizer.

All other authors confirm that there are no known conflicts of interest associated with this publication and there has been no significant financial support for this work that could have influenced its outcome.

We confirm that the manuscript has been read and approved by all named authors and that there are no other persons who satisfied the criteria for authorship but are not listed.

We further confirm that any aspect of the work covered in this manuscript that involved human patients has been conducted with the ethical approval of all relevant bodies and that such approvals are acknowledged within the manuscript.

\section{Acknowledgements}

The authors thank Bruce Fireman and Heather Whitaker for methodological input, Bart Spiessens for assistance with SAS code, 
and Judith Badoo and David Cho for facilitating collaboration. We also thank collaborators from the Vaccine Safety Datalink (VSD) (US) for their data contribution and methodological input.

The PRISM authors acknowledge the contributions of Joaquim Fernandes, Yihai Liu, Cheryl N. McMahill-Walraven, and Claire Spettell of Aetna, and Amy Ball, David Nau, Jane Stacy, and Yihua Xu of Humana.

\section{Appendix A. Global H1N1 GBS Consortium}

GBS Working Group: Antonio Addis, Aysha Akhtar, Judith Cope, Robert Davis, Paul Gargiullo, Xavier Kurz, Barbara Law, Grace Lee, Isabelle Sahinovic, and Jerry Tokars.

\section{Almeria (Spain): Pedro Serrano.}

Australia: The Victoria - Australia GBS Active investigators: Allen Cheng, Nick Andrews, Pat Charles, Hazel Clothier, Bruce Day, Timothy Day, Peter Gates, Richard MacDonnell, Les Roberts, Victoria Rodriguez-Casero, Tissa Wijeratne, Lynette Kiers, and the Paediatric Active Enhanced Disease Surveillance (PAEDS) network investigators: Christopher Blyth, Robert Booy, Elizabeth Elliott, Michael Gold, Helen Marshall, Peter McIntyre, Peter Richmond, Jenny Royle, Nicholas Wood and Yvonne Zurynski.

Barcelona (Spain): Gonzalo Calvo, Magda Campins, Nuria Corominas, Ferran Torres, Josep Valls, and Anna Vilella.

Department of Defense (US): Donald Dutra, Angelia Eick-Cost, Henry M Jackson Foundation working in support of the Armed Forces Health Surveillance Center, Patrick Garman, Zheng Hu, Henry M Jackson Foundation working in support of the Armed Forces Health Surveillance Center, and Marianne Rigo, Health Care Resolution Services working in support of the Military Vaccine Agency.

Food and Drug Administration (US): Judith Badoo, David Cho, Laura L. Polakowski, Sukhminder K. Sandhu, and Guoying Sun.

Hong Kong: Hoi-Shan Sophelia Chan, Kwok-yin Chan, Raymond Cheung, Yuk-fai Cheung, Sharon Cherk, SK Chuang, Joshua Waiming Fok, Bun-hey Fung, Kwai-fu Ko, Ka Wing Lau, Kwok-kwong Lau, Patrick Li, Hui-tung Liu, Shao-haei Liu, Tina Mok, Joanna So, Winnie Wong, and Shun-ping Wu.

Medicare (US): Armen Avagyan, Robert Ball, Dale Burwen, Riley L. Franks, Jonathan M. Gibbs, Rebecca E. Kliman, Garner F. Kropp, Thomas E. MaCurdy, David B. Martin, Laura L. Polakowski, Sukhminder K. Sandhu, Guoying Sun, and Christopher M. Worrall.

Mexico: Dra. Elvira Fuentes Fuentes, Paola Carolina Ojeda González, Jesus Reyna, and Rosa Patricia Vidal Vázquez.

Post-Licensure Rapid Immunization Safety Monitoring (PRISM) Network (US): Robert Ball, Martin Kulldorff, Grace Lee, Tracy A. Lieu, Richard Platt.

Quebec (Canada): Gaston De Serres.

Singapore: Kamilah Jabin, Bee Leng Sally Soh.

Vaccine Adverse Event Surveillance and Communication (VAESCO) Consortium (Europe): Lisen Arnheim-Dahlstrom, Anne Castot, Hester de Melker, Jeanne Dieleman, Jonal Hallgren, Bart Jacobs, Kari Johansen, Piotr Kramarz, Maryse Lapeyre, Tuija Leino, Ditte Molgaard-Nielsen, Mees Mosseveld, Henning K Olberg, Cormac Sammon, Christel Saussier, Martijn Schuemie, Agnes Sommet, Par Sparen, Henrik Svanstrom, Ann Vanrolleghem, and Daniel Weibel.

Valencia (Spain): Javier Diez Domingo (Vaccine Department,Center of Public Health Research(CSISP-FISABIO)), José Luís Micó Esparza (H. Arnau de Vilanova), Rafael M. Ortí Lucas (H. Clinico Universitario), Juan B. Mollar Maseres (H. Universitario La Fe), José Luís Alfonso Sánchez (Consorcio Hospital General Universitario), Mercedes Garcés Sánchez (H. De Manises), Vicente Zanón Viguer (H. Universitario Dr. Peset).
Department of Veterans Affairs (US): Francesca Cunningham, Bharat Thakkar, and Rongping Zhang.

\section{References}

[1] World Health Organization, Global Vaccine Safety Blueprint.WHO/IVB. 2012

[2] Mahoney RT, Maynard JE. The introduction of new vaccines into developing countries. IV: Global access strategies. Vaccine 2007;25(20):4003-11.

[3] Jacobson RM, Adegbenro A, Pankratz VS, Poland GA. Adverse events and vaccination-the lack of power and predictability of infrequent events in prelicensure study. Vaccine 2001;19(17-19):2428-33.

[4] Hua W, Sun G, Dodd CN, Romio SA, Whitaker HJ, Izurieta HS, et al. A simulation study to compare three self-controlled case series approaches: correction for violation of assumption and explanation of bias. Rockville, MD: Office of Biostatistics and Epidemiology, Center for Biologics Evaluation and Research, Food and Drug Evaluation; 2012. p. 22.

[5] Dodd C, Izurieta HS, Zuber P, Romio SA, Sturkenboom M, Hua W, Bonhoeffer J. International Collaborative Case Series Safety Monitoring for Pandemic 2009 H1N1 Vaccines: Estimation of the Risk of Guillain-Barre Syndrome. In: International conference on pharmacoepidemiology and therapeutic risk management. 2012

[6] Izurieta HS, Zuber BS, Dodd P, Bonhoeffer C, Sturkenboom J, Romio MS, et al. A proof-of-concept collaborative international study for the post-licensure epidemiological assessment of the safety of newly introduced vaccines. In: Vaccine safety update, brighton collaboration symposium. 2012

[7] Bonhoeffer J, Kohl K, Chen R, Duclos P, Heijbel H, Heininger U, et al. The Brighton Collaboration: addressing the need for standardized case definitions of adverse events following immunization (AEFI). Vaccine 2002;21(3-4):298-302.

[8] Garske T, Legrand J, Donnelly CA, Ward H, Cauchemez S, Fraser C, et al. Assessing the severity of the novel influenza A/H1N1 pandemic. BMJ 2009:339.

[9] Partridge J, Kieny MP. Global production of seasonal and pandemic (H1N1) influenza vaccines in 2009-2010 and comparison with previous estimates and global action plan targets. Vaccine 2010;28(30):4709-12.

[10] Whitaker HJ, Paddy Farrington C, Spiessens Bart, Musonda Patrick. Tutorial in biostatistics: the self-controlled case series method. Stat Med 2006;25(10):1768-97.

[11] Schonberger LB, Bregman DJ, Sullivan-Bolyai JZ, Keenlyside RA, Ziegler DW, Retailliau HF, et al. Guillain-Barré syndrome following vaccination in the national influenza immunization program: United States, 1976-1977. Am J Epidemiol 1979;110(2):105-23

[12] Sejvar JJ, Kohl KS, Gidudu J, Amato A, Bakshi N, Baxter R, et al. Guillain-Barre syndrome and Fisher syndrome: case definitions and guidelines for collection, analysis, and presentation of immunization safety data. Vaccine 2011;29(3):599-612.

[13] Farrington CP. Relative incidence estimation from case series for vaccine safety evaluation. Biometrics 1995;51(1):228-35.

[14] Dieleman J, Romio S, Johansen K, Weibel D, Bonhoeffer J, Sturkenboom M, et al. Guillain-Barre syndrome and adjuvanted pandemic influenza A (H1N1) 2009 vaccine: multinational case-control study in Europe. BMJ 2011:343:d3908.

[15] Salmon D, Yih WK, Lee G, Rosofsky R, Brown J, Vannice K, et al. Success of program linking data sources to monitor H1N1 vaccine safety points to potential for even broader safety surveillance. Health Aff (Millwood) 2012;31(11):2518-27.

[16] Nguyen M, et al. The Food and Drug Administration's Post-Licensure Rapid Immunization Safety Monitoring program: strengthening the federal vaccine safety enterprise. Pharmacoepidemiol Drug Saf 2012;21(Suppl. 1):291-7.

[17] Fine PE, Chen RT. Confounding in studies of adverse reactions to vaccines. Am J Epidemiol 1992;136(2):121-35

[18] Zhou W, Pool V, Iskander JK, English-Bullard R, Ball R, Wise RP, et al. Surveillance for safety after immunization: Vaccine Adverse Event Reporting System (VAERS) - United States, 1991-2001. MMWR Surveill Summ 2003;52(1):1-24.

[19] Maclure M, Fireman B, Nelson JC, Hua W, Shoaibi A, Paredes A, et al. When should case-only designs be used for safety monitoring of medical products? Pharmacoepidemiol Drug Saf 2012:21:50-61.

[20] Farrington CP, Whitaker HJ, Hocine MN. Case series analysis for censored, perturbed, or curtailed post-event exposures. Biostatistics 2009;10(1):3-16.

[21] DerSimonian R, Laird N. Meta-analysis in clinical trials. Control Clin Trials 1986;7(3):177-88

[22] Haber P, Destefano F, Anguly FJ, Iskander J, Shadomy SV, Weintraub $\mathrm{E}$, et al. Guillain-Barre syndrome following influenza vaccination. JAMA 2004:292(20):2478-81

[23] Crawford NW, Cheng A, Andrews N, Charles PG, Clothier HJ, Day B, et al. Guillain-Barre syndrome following pandemic (H1N1) 2009 influenza A immunisation in Victoria: a self-controlled case series. Med J Aust 2012;197(10):574-8

[24] Burwen DR, Sandhu SK, MaCurdy TE, Kelman JA, Gibbs JM, Garcia B, et al. Surveillance for Guillain-Barre syndrome after influenza vaccination among the Medicare population: 2009-2010. Am J Public Health 2012;102(10):1921-7.

[25] De Wals P, Deceuninck G, Toth E, Boulianne N, Brunet D, Boucher R, et al. Risk of Guillain-Barre syndrome following H1N1 influenza vaccination in Quebec. JAMA 2012;308(2):175-81.

[26] Greene SK, Rett M, Weintraub ES, Li L, Yin R, Amato AA, et al. Risk of confirmed Guillain-Barré syndrome following receipt of monovalent inactivated 
Influenza A (H1N1) and Seasonal Influenza Vaccines in the Vaccine Safety Datalink Project: 2009-2010. Am J Epidemiol 2012;175(11):1100-9.

[27] Wise ME, Viray M, Sevjar JJ, Lewis P, Baughman AL, Connor W, et al. Guillain-Barre syndrome during the 2009-2010 H1N1 influenza vaccination campaign: population-based surveillance among 45 million Americans. Am J Epidemiol 2012;175(11):1110-9.

[28] Yih WK, Lee GM, Lieu TA, Ball R, Kulldorff M, Rett M, et al. Surveillance for adverse events following receipt of pandemic 2009 H1N1 vaccine in the PostLicensure Rapid Immunization Safety Monitoring (PRISM) System: 2009-2010. Am J Epidemiol 2012;175(11):1120-8.

[29] Tokars JI, Lewis P, DeStefano F, Wise M, Viray M, Morgan O, et al. The risk of Guillain-Barre syndrome associated with influenza A (H1N1) 2009 monovalent vaccine and 2009-2010 seasonal influenza vaccines: results from self-controlled analyses. Pharmacoepidemiol Drug Saf 2012;21(5): 546-52.

[30] Bardage C, Persson I, Ortqvist A, Bergman U, Ludvigsson JF, Granath F. Neurological and autoimmune disorders after vaccination against pandemic influenza $A$ (H1N1) with a monovalent adjuvanted vaccine: population based cohort study in Stockholm, Sweden. BMJ 2011;343:d5956.

[31] Andrews N, Stowe J, Salman RA, Miller E. Guillain-Barre syndrome and H1N1 (2009) pandemic influenza vaccination using an AS03 adjuvanted vaccine in the United Kingdom: self-controlled case series. Vaccine 2011;29(45): $7878-82$.

[32] Salmon D, Proschan M, Forshee R, Gargiullo P, Bleser W, Burwen D et al. Association between Guillain-Barre syndrome and influenza A (H1N1) 2009 monovalent inactivated vaccines in the USA: a meta-analysis. Lancet 2013;381(9876):1461-8, http://dx.doi.org/10.1016/S0140-6736(12)62189-8.

[33] Gilca V, De Serres G, Hamelin ME, Boivin G, Ouakki M, Boulianne N, et al. Antibody persistence and response to 2010-2011 trivalent influenza vaccine one year after a single dose of 2009 AS03-adjuvanted pandemic H1N1 vaccine in children. Vaccine 2011;30(1):35-41.

[34] Stowe J, Andrews N, Wise L, Miller E. Investigation of the temporal association of Guillain-Barré syndrome with influenza vaccine and influenza like illness using the United Kingdom general practice research database. Am J Epidemiol 2009;169(3):382-8.

[35] Schonberger LB, Hurwitz ES, Katona P, Holman RC, Bregman DJ. Guillain-Barré syndrome: its epidemiology and associations with influenza vaccination. Ann Neurol 1981;9(S1):31-8.
[36] Hughes RAC, Rees JH. Clinical and epidemiologic features of Guillain-Barré syndrome. J Infect Dis 1997;176(Suppl. 2):S92-8

[37] Shui IM, Rett MD, Weintraub E, Marcy M, Amato AA, Sheikh SI, et al. Guillain-Barre syndrome incidence in a large United States cohort (2000-2009). Neuroepidemiology 2012;39(2):109-15.

[38] Weinberger B, Herndler-Brandstetter D, Schwanninger A, Weiskopf D, Grubeck-Loebenstein B. Biology of immune responses to vaccines in elderly persons. Clin Infect Dis 2008;46(7):1078-84.

[39] Hancock K, Veguilla V, Lu X, Zhong W, Butler EN, Sun H, et al. Cross-reactive antibody responses to the 2009 pandemic H1N1 influenza virus. N Engl J Med 2009;361(20):1945-52.

[40] Perez-Padilla R, de la Rosa-Zamboni D, Ponce de Leon S, Hernandez M, Quinones-Falconi F, Bautista E, et al. Pneumonia and respiratory failure from swine-origin Influenza A (H1N1) in Mexico. New Engl J Med 2009;361(7):680-9.

[41] Echevarría-Zuno S, Mejia-Arangure JM, Mar-Obeso AJ, Grajales-Muniz C, Robles-Perez E, Gonzalez-Leon M, et al. Infection and death from influenza A H1N1 virus in Mexico: a retrospective analysis. Lancet 2009;374(9707):2072-9.

[42] Abdulla S, Salim N, Machera F, Kamata R, Juma O, Shomari M, et al. Randomized, controlled trial of the long term safety, immunogenicity and efficacy of RTS,S/AS02D malaria vaccine in infants living in a malaria-endemic region. Malar J 2013;12(1):11

[43] Andrews N, Stowe J, Wise L, Miller E. Post-licensure comparison of the safety profile of diphtheria/tetanus/whole cell pertussis/haemophilus influenza type b vaccine and a 5-in-1 diphtheria/tetanus/acellular pertussis/haemophilus influenza type b/polio vaccine in the United Kingdom. Vaccine 2010;28(44):7215-20.

[44] Huang WT, Gargiullo PM, Broder KR, Weintraub ES, Iskander JK, Klein NP, et al. Lack of association between acellular pertussis vaccine and seizures in early childhood. Pediatrics 2010;126(2):263-9.

[45] Andrews N, Miller E, Waight P, Farrington P, Crowcroft N, Stowe J, et al. Does ora polio vaccine cause intussusception in infants? Evidence from a sequence of three self-controlled cases series studies in the United Kingdom. Eur JEpidemio 2001;17(8):701-6.

[46] Lasky T, Terracciano GJ, Magder L, Koski CL, Ballesteros M, Nash D, et al. The Guillain-Barre syndrome and the 1992-1993 and 1993-1994 influenza vaccines. N Engl J Med 1998;339(25):1797-802 